\title{
Maîtriser la conception des drones solaires à voilure souple : vers l'avènement des pseudo-satellites à hautes altitudes (HAPS)
}

\author{
Mastering the design of highly flexible solar powered UAV: towards the \\ concept of High Altitude Pseudo-Satellite (HAPS)
}

\author{
Bertrand Kirsch ${ }^{1}$, Olivier Montagnier ${ }^{1}$ \\ ${ }^{1}$ Centre de Recherche de l'Armée de l'Air, Ecole de l'air, Base Aérienne 701, 13661 Salon air, France, \\ bertrand.kirsch@ecole-air.fr, olivier.montagnier@ecole-air.fr
}

RÉSUMÉ. Initié dans les années 1950 avec l'invention des cellules photovoltaïques, le concept d'un drone solaire de haute altitude à endurance quasi-illimitée fait toujours l'objet d'une recherche active en raison de verrous technologiques persistants. La faible puissance propulsive extraite de la source solaire impose une architecture très particulière d'un tel aéronef avec notamment une voilure très allongée et très souple particulièrement vulnérable à des interactions destructrices. Ces dernières relèvent du domaine de l'aéroélasticité, discipline à l'interface entre l'aérodynamique et la mécanique des structures. Le présent article illustre ainsi l'approche nécessairement transdisciplinaire de ce défi technologique et présente des solutions innovantes issues de la recherche. Celles-ci devraient à terme permettre l'exploitation commerciale de ce concept capable de combler dans le domaine de l'observation et des télécommunications un creux capacitaire entre les actuels drones conventionnels de haute altitude et les solutions satellitaires.

ABSTRACT. Initiated in the 1950s with the invention of photovoltaic cells, the almost unlimited endurance high altitude solar drone concept is still under heavy development because of persistent technological barriers. The low propelling power extracted from solar sources involves a specific design of an airframe consisting of a very lightweight and flexible airframe particularly vulnerable to destructive interactions. The latter fall within the scope of aeroelasticity, a discipline between aerodynamic and structural mechanics. This article illustrates the transdisciplinary approach required to achieve such a challenging goal and presents innovative solutions based on research work. These should enable commercial exploitation of such a concept and then fill the gap between conventional HALE drones and satellites in the field of observation and telecommunication.

MOTS-CLÉS. Drone HALE, HAPS, énergie solaire, aéroélasticité, flutter, endurance.

KEYWORDS. HALE UAV, HAPS, solar energy, aeroelasticity, flutter, endurance.

\section{Introduction}

L'histoire de l'aviation est jalonnée depuis ses débuts de records successifs qui ont permis, étape par étape, d'améliorer les performances des plus lourds que l'air. Qu'il s'agisse de la distance franchissable, de la vitesse de vol ou encore de l'altitude de croisière, la rencontre de progrès techniques et de pionniers prêts à les mettre en œuvre a repoussé au fil des années les limites de l'aviation. Parmi ces limites, l'endurance soulève un certain nombre de défis pour les chercheurs comme pour les concepteurs d'aéronefs.

L'endurance d'un aéronef est en soi une notion assez simple : il s'agit de la durée qui s'écoule entre le moment où les roues (quand il y en a) quittent le sol et celui où le contact avec la terre ferme s'établit à nouveau. Cette endurance est directement liée à la quantité et au type d'énergie disponible pour la motorisation. Les énergies fossiles comme le kérosène sont quasiment les seules utilisées en aéronautique en raison de leurs grandes densités énergétiques (figure 1). L'inconvénient majeur de cette solution est qu'elle ne permet pas de régénérer même partiellement la quantité d'énergie embarquée au départ sauf à avoir recours au ravitaillement en vol. Cette technique, fondamentale en aéronautique militaire, revêt toutefois aux moins deux limitations importantes pour ce qui est de 
l'optimisation de l'endurance. D'une part elle ne permet pas d'augmenter l'endurance intrinsèque d'un aéronef et reporte ainsi le « problème » sur l'avion ravitailleur. D'autre part elle contraint les aéronefs ravitailleurs et ravitaillés à être capable de voler dans les mêmes conditions, notamment de vitesse et d'altitude.

L'électricité ou l'hydrogène peuvent être des alternatives au kérosène. Dans le cas d'un aéronef, l'électricité est stockée dans des batteries et utilisée directement par un ou plusieurs moteurs électriques faisant tourner une ou plusieurs hélices (e.g. l'E-Fan d'Airbus ayant volé en 2014 [JUV 16]). L'hydrogène est quant à lui utilisé soit comme carburant dans un moteur à hydrogène (e.g. le Tupolev Tu-155 ayant volé en 1989), soit comme source d'énergie pour une pile à combustible générant de l'électricité en replacement de la batterie dans la solution électrique (e.g. l'ENFICA-FC développé par Politecnico di Torino [ROM 13]). Cet hydrogène est stocké dans des réservoirs sous très haute pression pour minimiser son volume. Malheureusement ces différentes solutions alternatives, pour intéressante qu'elles soient d'un point de vue écologique, ne répondront pas au problème d'endurance car elles présentent une densité énergétique moindre que celle du kérosène (figure 1).

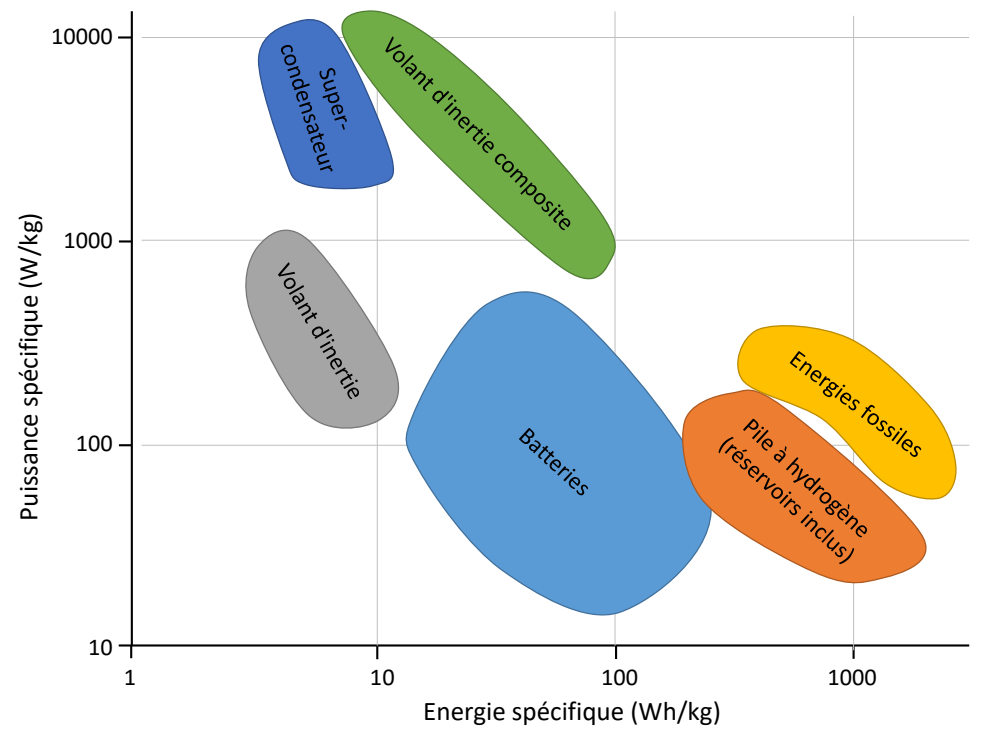

Figure 1. Comparaison des sources d'énergie disponibles pour les drones (diagramme de Ragone): puissance spécifique versus masse spécifique en échelle logarithmique

L'énergie solaire semble être l'unique solution pour résoudre la limitation de l'endurance. Cette énergie est illimitée le jour pour un aéronef volant au-dessus de la couverture nuageuse, c'est-à-dire, au-dessus de la troposphère (comprise entre 0 et $13 \mathrm{~km}$ ). Le principe de fonctionnement est alors assez simple. Le jour, des cellules photovoltaïques positionnées à la surface de l'aéronef récupèrent l'énergie nécessaire au vol et aux instruments à laquelle il faut ajouter celle nécessaire au vol de nuit. Cette dernière est stockée soit dans des batteries soit sous forme d'hydrogène en utilisant une pile à combustible réversible (dite aussi régénérative). La nuit, l'énergie stockée est alors restituée à la propulsion et aux instruments. Un tel concept, lorsqu'il est mis en œuvre dans un système de drone afin d'éviter des limitations en temps de vol d'équipage, adosse la limite d'endurance non plus à son autonomie en énergie embarquée mais à la durée allouée entre deux visites de maintenance, pouvant ainsi aller jusqu'à plusieurs années. Cette optimisation poussée à l'extrême de drone HALE (Haute Altitude Longue Endurance) solaire est une implémentation technologique du concept plus général de pseudo-satellite à hautes altitude (High Altitude Pseudo-Satellite - HAPS). Ces solutions sont étudiées depuis le milieu des années 1980. Pourtant, à ce jour, aucun appareil de ce type n'est exploité.

L'objectif de cet article est d'analyser les difficultés de conception de ces drones HALE solaire pour expliquer le temps écoulé entre la création du concept, sa mise en œuvre et peut-être un jour son exploitation et donc l'aboutissement du processus d'innovation. Pour cela, nous allons tout d'abord 
présenter les applications et les enjeux capacitaires puis l'historique des projets, des records et des accidents de l'aéronautique solaire. Nous verrons ensuite les verrous technologiques persistants qui n'ont pas permis à ce jour la concrétisation pleine et entière de ce concept d'aéronef. Nous montrerons en particulier la nécessité de concevoir une voilure composite souple et les conséquences physiques qui résulte de cette nécessité comme l'instabilité de flottement (dite aussi flutter). Enfin, parmi les voies de progrès possibles qui permettraient de trouver une solution viable, nous évoquerons une idée directement issue de la recherche : le tissage aéroélastique.

\section{Enjeux capacitaires : observation et télécommunications}

Le potentiel des HAPS est important car il s'agit surtout d'étendre l'emploi du vecteur aérien dans un domaine capacitaire jusqu'alors réservé aux satellites. Il n'est pas ici question de remplacer globalement un moyen par un autre, mais plutôt de combler un creux capacitaire d'où leur qualificatif de pseudo-satellites. Là où la mise en place d'un moyen spatial lourd sera peu efficient et manquera de réactivité, la mise en œuvre d'un drone de haute altitude, dusse-t-il mettre quelques dizaines d'heures à arriver sur zone, prendrait tout son sens, à condition qu'il puisse ensuite assurer la permanence sur zone. Deux applications principales qui concernent à la fois les utilisateurs civils et militaires se distinguent : l'observation et les télécommunications.

En effet, en volant à haute altitude et avec une endurance extrêmement grande, ces aéronefs n'ont pas d'équivalent. Les altitudes visées (une vingtaine de kilomètres) sont de l'ordre du plafond de croisière de l'avion de reconnaissance Lockheed U-2 et sont idéales pour faire de l'observation (figure 2). Dans cette zone, le drone sera peu vulnérable et situé dans un espace totalement libre de trafic aérien, les avions de lignes croisant aux alentours de $12 \mathrm{~km}$ d'altitude. Technologiquement, l'intérêt de ces altitudes est de se placer au-dessus des nuages mais aussi de diminuer l'atténuation atmosphérique de l'énergie solaire et de se situer dans une zone de vent faible (facilitant la permanence sur zone). En revanche, la très faible densité de l'air est très défavorable à la sustentation de l'appareil.

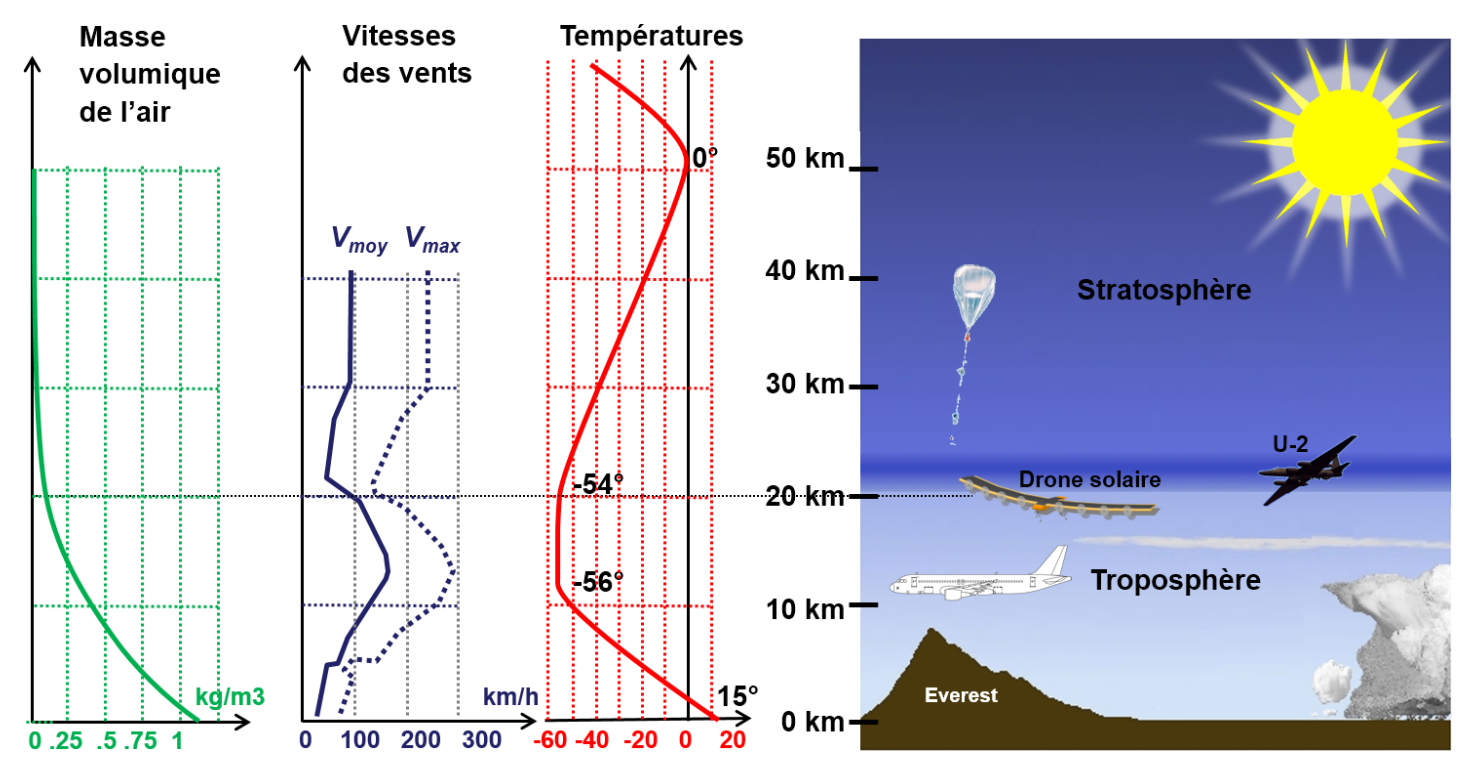

Figure 2. Positionnement d'un HAPS dans l'atmosphère

A $20 \mathrm{~km}$ d'altitude, malgré la rotondité de la terre, un HAPS pourrait émettre sur un rayon de 200 $\mathrm{km}$. Avec un émetteur adapté, seulement 4 à 5 plateformes pourraient couvrir l'ensemble du territoire français (figure 3). D'après Cestino [CES 06], un HAPS de ce type remplacerait cinq drones MALE (Moyenne Altitude Longue Endurance) à énergie conventionnelle pour un coût quatre fois plus faible (750 euros par heure de vol contre 3060 euros par heure de vol). 
La NASA a recensé les applications potentielles dans les différents domaines. Dans les sciences de la terre, il est estimé que ce type de drone pourrait servir pour la météorologie, l'observation océanique et géologique, et la surveillance des polluants atmosphériques ainsi que la couche d'ozone. En ce qui concerne les applications de défense et de sécurité ces altitudes permettraient de faire des relais de télécommunication appliqués à la surveillance des frontières, des zones maritimes, du transport illicite de matériaux radioactifs, du suivi des incendies de forêt, etc. Enfin en termes d'applications commerciales autres que le relais de télécommunication, ces drones pourraient être utilisés dans le suivi des transports routiers, des ressources naturelles, le relevé topographique et l'agriculture de précision.

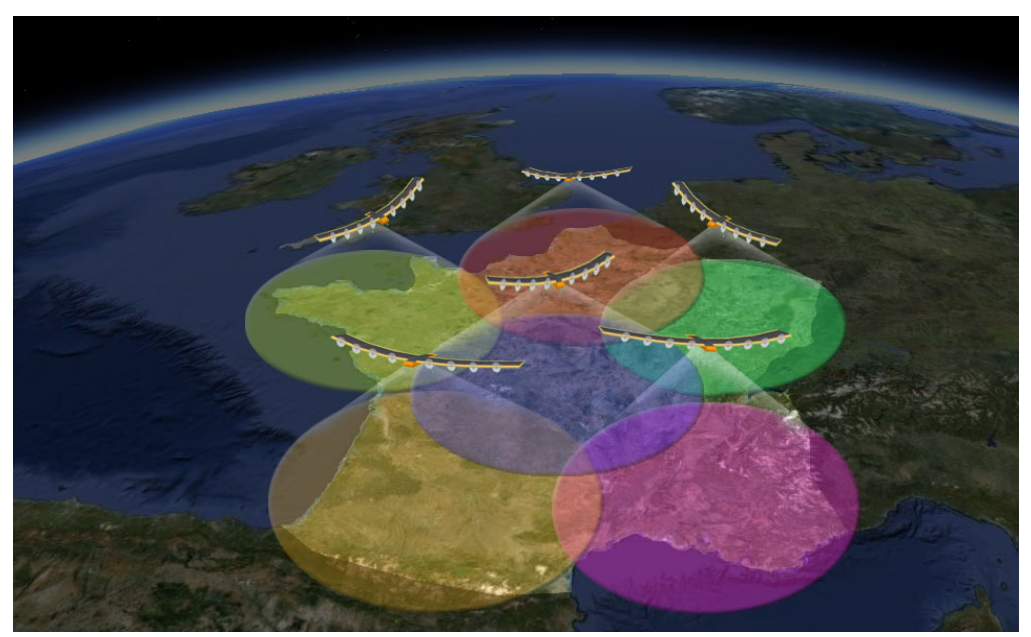

Figure 3. Cinq drones HALE solaires positionnés à 20 km d'altitude pourraient couvrir l'ensemble de la France

\section{Historique du vol solaire : projets, records et accidents}

Les cellules photovoltaïques sont découvertes en 1954. La même année, August Raspet propose d'installer ces cellules sur l'extrados d'un planeur et d'utiliser une propulsion électrique. L'idée du vol solaire est née, marquant ainsi le début d'une longue phase de maturation technologique.

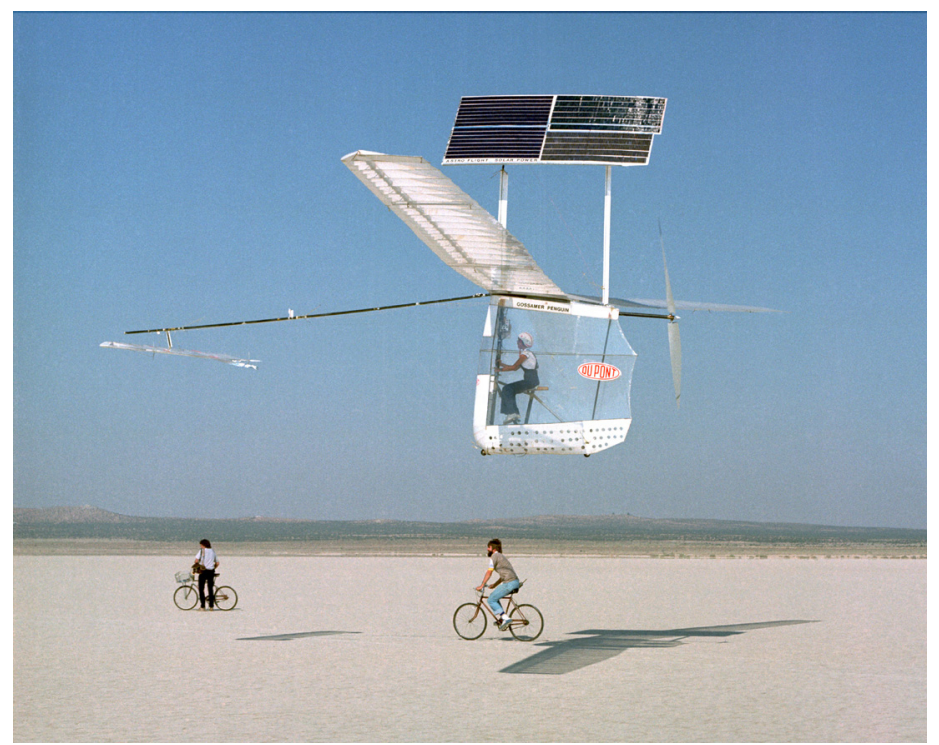

Figure 4. Gossamer Penguin durant des essais en 1980

En 1974, le drone Sunrise I, d'une envergure de 9,7 m et d'une masse de $12 \mathrm{~kg}$, réalise le premier vol solaire en Californie. En 1975, son successeur le Sunrise II atteint l'altitude record de 5,2 km [BOU 85]. Le développement des premiers drones solaires est alors étroitement lié au développement des avions solaires tels que Solar One (GB), Solar Riser (US) et Solair I (DE). Le Gossamer Penguin 
développé par AeroVironment (US) sera le premier avion piloté volant uniquement à l'énergie solaire (figure 4). Il effectuera un vol de $3 \mathrm{~km}$ en Californie le 7 août 1980. De ce projet naît le Solar Challenger. Il possède une envergure de 14,3 $\mathrm{m}$ et une masse à vide de seulement $90 \mathrm{~kg}$ [MAC 83]. Emportant une plus grande quantité de cellules sur les ailes et sur le plan arrière, cet avion uniquement solaire sera le premier à pouvoir parcourir de grandes distances. Il traverse la Manche en 5 heures et 23 minutes, le 7 juillet 1981. L'ensemble de ces projets montre l'importance des enjeux de masses qu'implique la grande faiblesse de l'énergie disponible. Ils sont construits à l'aide de matériaux composites et entoilés par des polymères extra légers (figure 4).

Pathfinder (1981-1997)

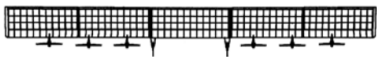

Pathfinder Plus (1997-1998)

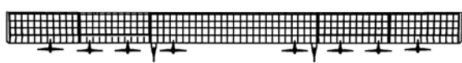

Centurion (1996-1998)

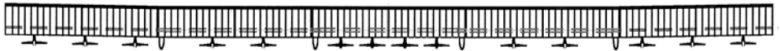

Helios Prototype (HP01), High-Altitude Configuration (1998-2002)

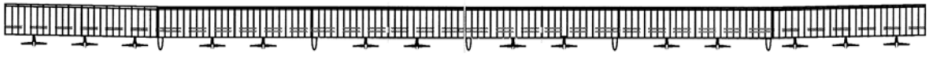

Helios Prototype (HP03), Long-Endurance Configuration (2003)

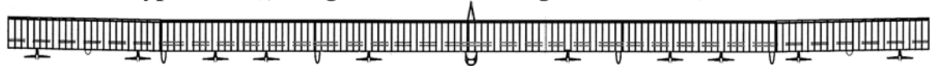

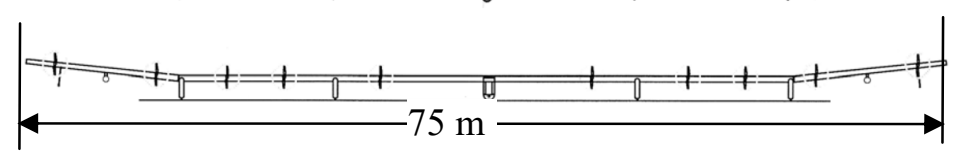

(a)

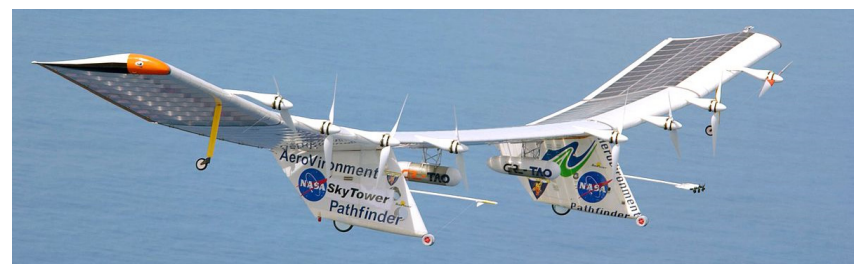

(b)

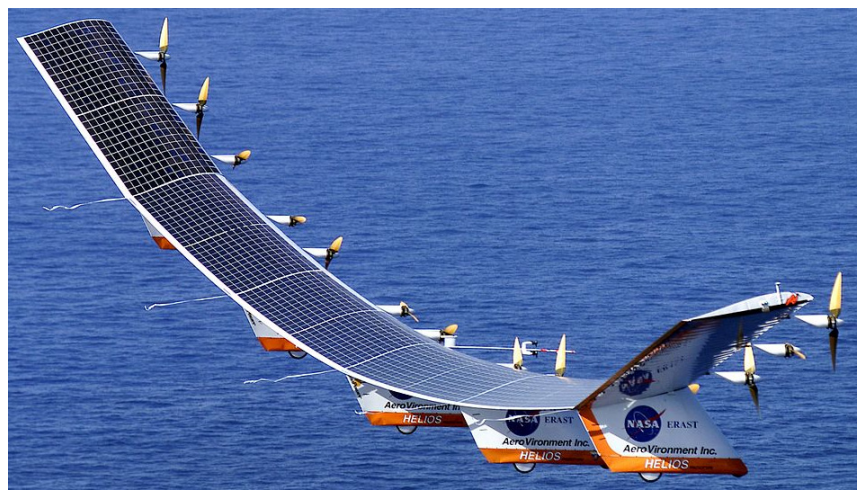

(c)

Figure 5. Programme ERAST de la NASA : a) comparaison des différents drones solaires du programme b) Pathfinder plus en juin 2002 c) Helios en juillet 2001.

Au début des années 90, la NASA lance le premier grand programme de drone HALE solaire appelé ERAST (figure 5a). L'objectif était de construire un drone capable de voler sans interruption durant six mois au-delà de $18 \mathrm{~km}$ d'altitude. Le drone Pathfinder fait ses premiers vols dès 1994 [FLI 98]. Il avait en fait été construit en 1983 par AeroVironment lors d'un projet classé secret défense. Pathfinder atteint l'altitude record de 15,4 km au-dessus de la Californie le 11 septembre 1995. A partir de 1997, les essais sont réalisés à Hawaii notamment pour les conditions favorables d'ensoleillement et de prévisibilité météorologique. Il obtient alors le record d'altitude pour un avion propulsé par des hélices $(21,8 \mathrm{~km})$. Le rendement des cellules n'est alors que de $14 \%$ et les petites batteries ne permettent pas le vol de nuit. En 1998, Pathfinder devient Pathfinder Plus (figure 5a et figure 5b). La partie centrale de la voilure du drone a été allongée et deux moteurs ont été ajoutés. La puissance potentielle disponible passe de $7500 \mathrm{~W}$ à $12500 \mathrm{~W}$. Il établit un nouveau record à $24,4 \mathrm{~km}$ le 6 août 1998, le vol dura près de 15 h. En 2001, Pathfinder Plus fut employé à démontrer sa capacité à effectuer une mission de contrôle de l'irrigation de la plus grande plantation de café des Etats-Unis sur l'île de Kauai [HER 02]. Sa charge utile est alors de $45 \mathrm{~kg}$. En 1998, le drone Centurion (figure 5a) est construit et préfigure le drone Helios qui sera équipé d'une pile à combustible réversible permettant le vol de nuit. Ce dernier fera ses premiers vols en 1999 (Figs. 5a et 5c). Il possède une envergure de $75 \mathrm{~m}$, supérieure à celle d'un Boeing 747 pour un poids total inférieur à $930 \mathrm{~kg}$. Sa voilure est recouverte de 62000 cellules photovoltaïques et sa charge utile est de l'ordre de $100 \mathrm{~kg}$. Sa vitesse est de seulement $35 \mathrm{~km} / \mathrm{h} \mathrm{au}$ niveau de la mer, en revanche elle est bien supérieure à $100 \mathrm{~km} / \mathrm{h}$ à haute altitude et peut atteindre 270 $\mathrm{km} / \mathrm{h}$ à très haute altitude. Helios atteint 29,5 km d'altitude le 13 août 2001. Malheureusement, lors de son second vol en configuration longue durée (i.e. avec pile à combustible), le 26 juin 2003, le drone 
rencontre une oscillation instable qui lui sera fatal (figure 6). La commission d'enquête conclut à l'apparition d'un mode d'oscillation en tangage couplé avec un mode structural [NOL 04]. Autrement dit, le drone s'est mis à battre des ailes alors qu'il n'était évidemment pas prévu pour cela. Cet échec met fin au programme ERAST (97 millions de dollars principalement utilisés pour le développement des drones solaires) avant de pouvoir évaluer le vol de longue durée.

Durant la dernière décennie, plusieurs projets de drones HALE solaires mais aussi d'avions solaires ont vu le jour. En Europe, le projet le plus abouti est celui du drone Zephyr (22,5 m d'envergure pour $53 \mathrm{~kg}$ ) développé par Qinetiq puis racheté par Airbus Defense \& Space (version Zephyr S sur la figure 7). Ce drone est relativement petit et sa charge utile n'est que de $2,5 \mathrm{~kg}$. Ce dernier est actuellement le détenteur du record d'endurance avec 14 jours consécutifs en vol (336 heures 22 minutes et 8 secondes du 9 au 23 juillet 2010). A titre de comparaison, le record en vol habité sans ravitaillement est détenu par le Rutan Voyager en 216 heures 6 minutes et 43 secondes du 13 au 23 décembre 1986, talonné par le Solar Impulse 2 qui réalise la performance sans énergie fossile durant 117 heures et 51 minutes du 28 juin au 3 juillet 2015. Il est à noter que Solar Impulse 2 est capable de réaliser des vols continus (jours et nuits) mais avec une météo idéale (peu de nuages) car son plafond en croisière est de $8500 \mathrm{~m}$.
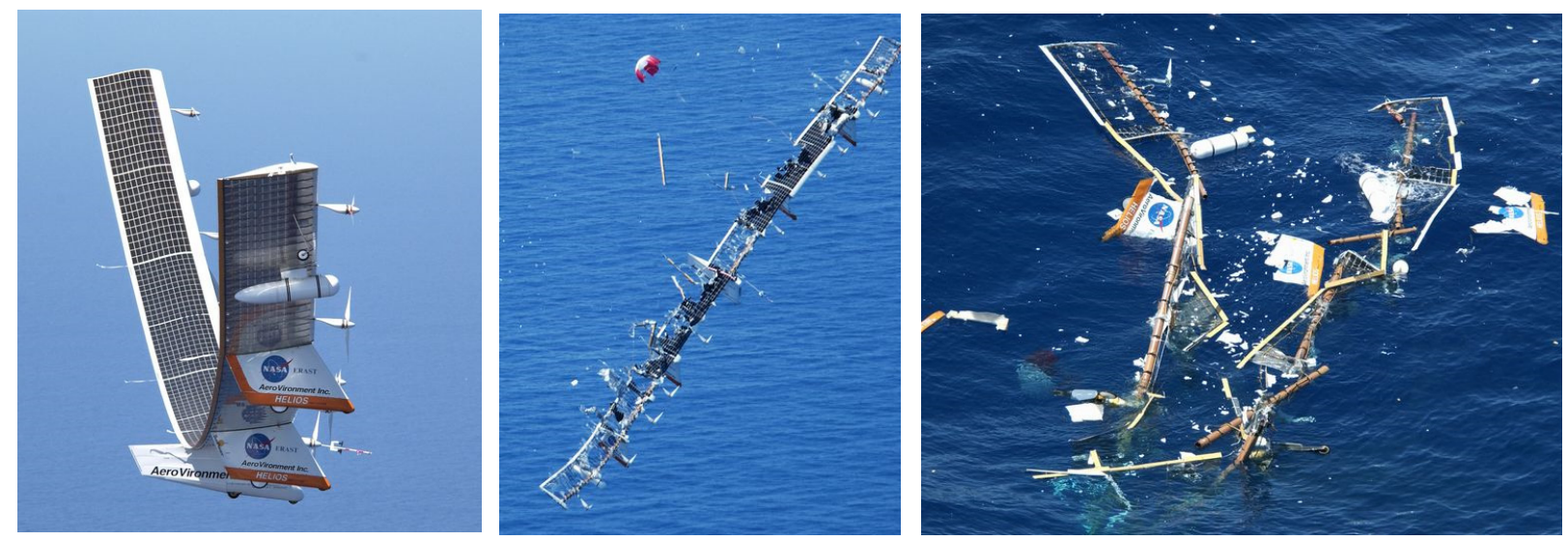

Figure 6. Accident de Helios le 26 juin 2003 (photos prisent par l'hélicoptère suiveur)

Aux États-Unis, trois grands projets de drones HALE solaires ont été lancé avec plus ou moins de succès. Le premier est l'appel à projet Vulture lancé par la Darpa en 2008 à hauteur de 90 millions de dollars. L'objectif était de transférer aux industriels le savoir acquis par le programme ERAST. Le cahier des charges est extrêmement ambitieux puisqu'il devait permettre la réalisation d'un prototype capable de voler cinq ans sans interruption. En 2010, Boeing remporte la première phase de l'appel à projet avec son drone SolarEagle face à Lockeed Martin et Aurora Flight Sciences. Malheureusement, le projet a été abandonné en 2012. Un autre grand projet est le drone Aquila de la société Facebook (30 m d'envergure pour $400 \mathrm{~kg}$ ) développé par son Connectivity Lab et construit par la société Ascenta en Angleterre (figure 7). L'objectif pour la société est de pouvoir distribuer internet dans les zones isolées (sur un rayon de $80 \mathrm{~km}$ autour du drone). L'originalité de cet appareil est d'être positionné à haute altitude par des ballons d'hélium avant de commencer à voler puis d'atterrir au bout de trois mois en glissant sur de l'herbe. Le drone effectue un premier vol le 28 juin 2016 mais s'écrase à l'atterrissage, cette fois-ci en raison d'une interaction entre les commandes de vol automatiques et la souplesse de l'aile volante [NTS 17a]. Après l'accident, le prototype a été modifié et a permis de faire en 2017 un nouveau vol de 46 minutes. Enfin, le troisième projet est le drone Solara $50(50 \mathrm{~m}$ d'envergure et $15 \mathrm{~m}$ de long) de la start-up Titan Aerospace rachetée par la société Google en avril 2014 (figure 7). Le prototype à échelle réduite s'écrase lors de son premier vol, le 1er mai 2015, peu après le décollage. Le rapport d'enquête conclu à une vitesse excessive déclenchée par des courants ascendants forts et une grande déformation de la voilure conduisant à une rupture en vol de l'aile gauche [NTS 10b]. 
Enfin, en Chine, la China Aerospace Science and Technology Corporation (CASC) a réalisé un prototype de drone HALE solaire appelé Rainbow (45 m d'envergure). Il a réalisé le 24 mai 2017 un vol de 15 heures et a atteint une altitude de $20000 \mathrm{~m}$.

Un historique plus complet sur les drones HALE solaire est donné dans [NOT 08] et [MON 13].

Le concept d'un plus lourd que l'air à endurance illimitée, du fait des efforts de recherches encore importants à consentir et des nombreux accidents, est concurrencé par une seconde formule mettant en œuvre des dirigeables de haute altitude. Pour illustrer cette course, citons la société Google qui a abandonné le projet de drone HALE solaire Solara 50 pour se consacrer au développement d'une solution à base d'aérostat. Les efforts de la compagnie sont désormais concentrés sur le projet de ballons stratosphériques Loon destinés à assurer la couverture réseaux des zones non desservies. Ces ballons font 15 mètres de diamètre, volent à 20 kilomètres d'altitudes et ont un objectif d'endurance fixé à 187 jours. Ils ont d'ailleurs été utilisés en octobre 2017 pour rétablir une couverture réseaux d'urgence aux habitants de Porto Rico après le passage de l'ouragan Maria. L'inconvénient majeur de cette solution est d'être tributaire des vents pour se diriger, le seul paramètre de commande étant le changement d'altitude.

Autre candidat plus léger que l'air de cette course à l'endurance, le projet Stratobus de la société Thales Alenia Space, dirigeable de 100 mètres de long prévu pour se placer à 20 kilomètres d'altitude [BAU 15]. Cette plateforme présente l'avantage d'être motorisée par une solution électrique alimentée par une pile à combustible réversible, ce qui la rend repositionnable contrairement à un ballon stratosphérique. L'endurance annoncée est de 5 années, le premier vol est attendu en 2019 pour une mise en service en 2020.

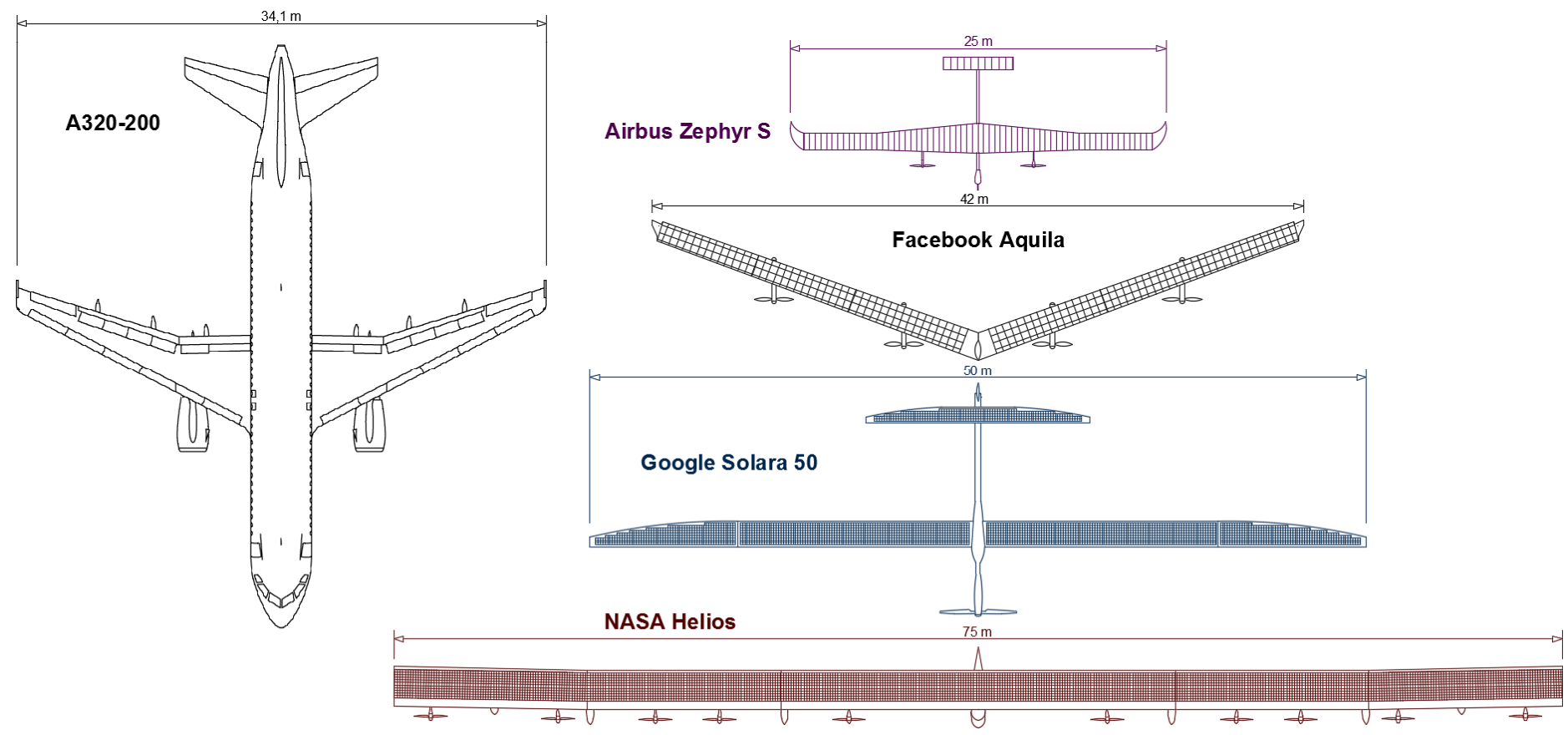

Figure 7. Comparaison des différentes géométries de drones solaires avec un Airbus A320-200 (vue de dessus).

\section{Résolution d'un paradoxe scientifique et technique}

L'éclairage de ce rappel historique du vol solaire fait ressortir l'existence d'un paradoxe scientifique et technique. En effet, malgré les investissements étatiques et privés conséquents déjà réalisés dans le domaine, le concept d'un drone solaire à endurance illimitée n'a toujours pas abouti alors même qu'il met en œuvre des technologies qui ont toutes réalisées individuellement des progrès considérables au 
cours des dernières décennies. Afin de résoudre ce paradoxe, la suite de l'exposé s'intéresse aux verrous technologiques intrinsèques au concept de drone solaire.

\subsection{L'énergie solaire : illimitée?}

La première question à se poser dans le dimensionnement d'un système ne fonctionnant qu'à l'énergie solaire est celle de sa disponibilité en quantité suffisante. Cette énergie est illimitée le jour et il suffit d'utiliser des cellules photovoltaïques pour la récupérer. Ce n'est malheureusement pas si simple. La quantité d'énergie que l'on va récupérer va dépendre directement de la surface de cellules et de leur rendement, mais aussi de la position du drone sur le globe et de la date à laquelle il s'y trouve. En effet l'énergie récupérée dépend directement de l'angle que font les rayons lumineux avec les cellules (a priori à l'horizontale). La figure 8 montre clairement que la puissance disponible dans une journée à nos latitudes est très dépendante du jour de l'année et est somme toute très faible. A titre d'exemple, la puissance captée à midi au solstice d'hiver par un mètre carré de cellules ayant un rendement de 19\% (correspondant à celles disposées sur Helios) est de $80 \mathrm{~W}$. En particulier, l'énergie par mètre carré captable dans une journée, c'est-à-dire la surface sous la courbe noire, est cinq fois plus faible au solstice d'hiver qu'au solstice d'été.

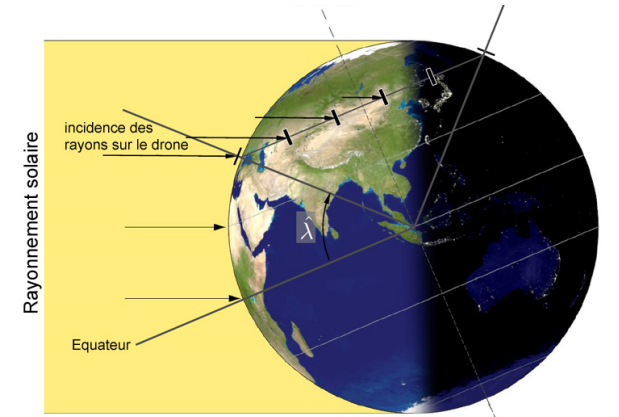

(a)

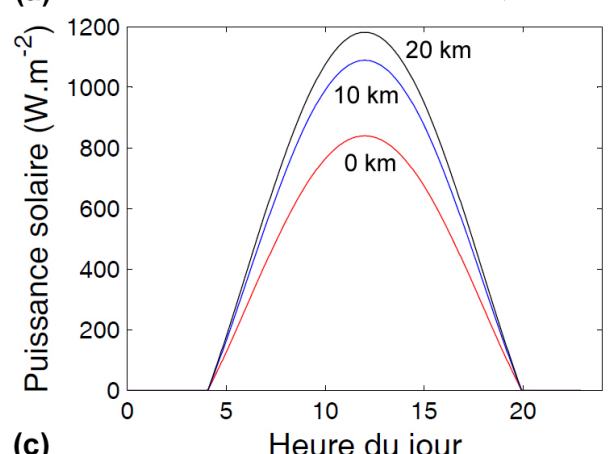

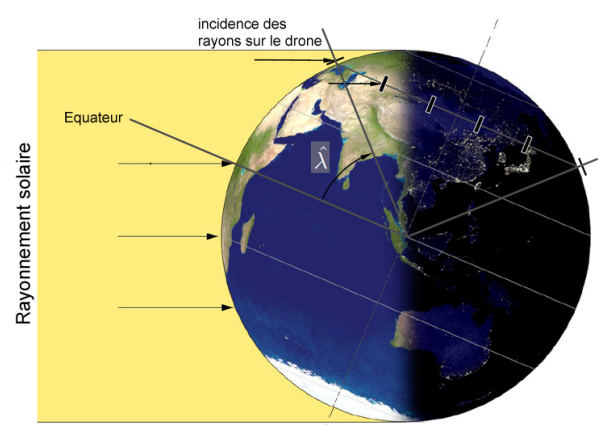

(b)

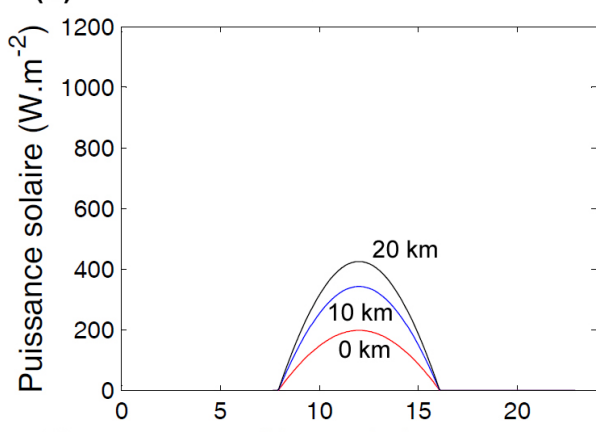

(d)

Figure 8. Ensoleillement d'un drone HALE solaire (a) au solstice d'été et (b) au solstice d'hiver et puissance solaire surfacique disponible à l'horizontale en fonction de l'altitude, à $48^{\circ}$ de latitude (Paris), (c) au solstice d'été et (d) au solstice d'hiver

Imaginons maintenant que l'on désire faire voler un drone conventionnel avec la seule énergie solaire. Prenons l'exemple d'un drone d'observation HALE tel que le « Global Hawk » possédant une masse totale de l'ordre de $10 \mathrm{t}$ et évoluant à $640 \mathrm{~km} / \mathrm{h}$ à $20 \mathrm{~km}$ d'altitude. Maintenir ce drone en vol à son altitude de croisière demande une puissance propulsive d'environ $610 \mathrm{~kW}$. Pour obtenir cette puissance le jour du solstice d'été à midi et à nos latitudes, il faudrait, en supposant que $20 \%$ de l'énergie solaire recueillie soit convertie en puissance motrice, plus de $2600 \mathrm{~m}^{2}$ de cellules solaires soit l'équivalent d'un demi stade de football. Au solstice d'hiver et à midi, cette surface atteindrait près de $7200 \mathrm{~m}^{2}$. Cette dernière serait encore très largement amplifiée si le drone devait continuer à voler la nuit car une grande partie de l'énergie devrait être stockée dans des batteries. En pratique la surface de la voilure du « Global Hawk » n'est que d'environ $50 \mathrm{~m}^{2}$. 
Cette démonstration permet de conclure rapidement qu'un drone solaire doit : maximiser la surface de cellules ; utiliser des cellules ayant un rendement élevé mais pour une masse surfacique faible ; consommer excessivement peu d'énergie.

\subsection{Les clés de l'endurance}

Revenons maintenant à la notion d'endurance. Du point de vue aérodynamique d'abord, le paramètre clé est la finesse. Il s'agit du rapport entre l'effort de portance et l'effort de trainée. Le type d'aéronef qui optimise ce paramètre est le planeur de compétition qui peut dépasser les 70 en conditions idéales. En pratique, cela signifie qu'en air calme un tel planeur peut se déplacer horizontalement de 70 kilomètres avant d'atterrir en partant d'une altitude d'un kilomètre.

Du point de vue structural ensuite, il faut construire une structure la plus légère possible pour deux raisons principales. La première est de limiter autant que possible la puissance propulsive qui sert à compenser les pertes dues à la traînée aérodynamique. En effet, plus la structure est légère, plus la vitesse d'équilibre permettant de maintenir l'altitude sera faible et plus la traînée sera faible. La deuxième est de dégager le plus de masse disponible possible pour embarquer des capacités de stockage d'énergie et de la charge utile (capteurs, relais,...).

Les points évoqués supra nous permettent de définir le portrait-robot d'un aéronef à endurance maximale. Une aile volante pour maximiser la surface des cellules solaires, ressemblant à une voilure de planeur pour optimiser la finesse, volant à haute altitude pour maximiser l'énergie captée et construite en matériaux composites type fibre de carbone/résine époxy pour allier résistance et légèreté [MON 09 ; MON 10]. Les exemples de la partie 3 respectent quasiment tous cette formule.

Le raisonnement conduisant à la définition d'un aéronef à endurance maximale et les technologies associées semble déjà maîtrisés dans le monde aéronautique, la part des matériaux composites dans la fabrication des aéronefs par exemple est aujourd'hui de plus en plus importante dans l'aéronautique militaire et civile. Elle dépasse les 50\% en masse sur l'Airbus A350 et le Boeing 787.

Pourquoi dans ce contexte aucun drone HALE solaire n'a pu démontrer sa capacité à se maintenir en l'air pour une durée illimitée?

\subsection{Un verrou technologique : l'aéroélasticité des voilures souples}

Quels que soient les progrès actuels et attendus des cellules photovoltaïques, la réponse est à chercher dans toutes les disciplines concourant à la conception d'un aéronef pour espérer atteindre un jour une endurance illimitée. Le paradoxe évoqué précédemment réside dans le fait que l'optimisation du tout n'est pas équivalente à l'optimisation de ses parties. En effet, si notre solution est optimisée du point de vue de l'aérodynamique d'une part et de la conception de la structure d'autre part, elle se révèle peu performante à la frontière entre les deux. Cette frontière, appelée aéroélasticité étudie les mécanismes d'interaction entre les efforts aérodynamiques appliqués à l'aéronef et l'élasticité de sa structure.

Des ailes à grand allongement et très légères sont nécessairement très souples et cette souplesse est un facteur de vulnérabilité vis-à-vis d'interactions destructrices, la plus connue d'entre elles étant l'instabilité de flottement ou «flutter » (de l'anglais battement d'ailes). Ce phénomène de flutter est à la fois bien connu de la communauté aéronautique et source de nombreuses explications erronées. Une explication précise du phénomène est donnée dans l'encadré 1 accompagnée d'un historique dans l'encadré 2. Ces phénomènes d'interactions, de mieux en mieux maîtrisés à la fin du XXème siècle, font leur retour sur le devant de la scène aéronautique avec l'exploration de la limite d'endurance. Ce phénomène est à l'origine des accidents du drone Helios de la NASA et du drone Aquila de Facebook (partie 3). L'accident du drone Solara 50 de Google est quant à lui issu d'un phénomène proche car lié à la souplesse de la voilure. 


\section{Le flutter qu'est-ce que c'est?}

Concrètement on parle de flutter lorsqu'une surface portante d'un avion (aile ou empennages) va dans certaines conditions de vol subir un mouvement oscillant couplé de flexion (battement de l'aile) et de torsion. Ce mouvement s'amplifie de manière très rapide menant dans le meilleur des cas un mouvement stabilisé de forte amplitude et dans le pire des cas à la destruction brusque de l'aéronef. Le déclenchement de cette instabilité est associé à une vitesse excessive, c'est d'ailleurs un des éléments qui détermine la vitesse de vol à ne pas dépasser pour un aéronef donné.

Ce phénomène est souvent décrit comme étant une résonance, or ce n'est pas le cas, démontrons le. En mécanique on parle de résonance lorsqu'une excitation périodique d'entrée est amplifiée de manière importante par une structure. Les exemples sont nombreux, on peut citer le cas d'une troupe marchant au pas sur un pont qui risque de faire entrer ce dernier en résonance si la fréquence du pas correspond à la fréquence propre du pont. Par conséquent, qui dit résonance dit excitation périodique, élément absent du phénomène de flutter : nul besoin de subir des rafales de vent à une fréquence donnée pour déclencher un tel phénomène, il suffit pour une altitude et un nombre de Mach donnés de dépasser une certaine vitesse de vol.

Après avoir vu ce que le flutter n'est pas, décortiquons le mécanisme qui conduit à son apparition. Il s'agit comme évoqué précédemment d'une interaction entre l'écoulement d'air et l'élasticité de la structure, interaction sur deux aspects.

La première interaction concerne les fréquences propres d'oscillation de la voilure. Au sol, de la même manière qu'un diapason excité par un choc va osciller à la fréquence du la à $440 \mathrm{~Hz}$, une aile excitée en flexion va battre à une fréquence de quelques $\mathrm{Hz}$ tandis qu'une excitation en torsion déclenche une oscillation pivotante d'une fréquence supérieure. En vol, l'interaction avec l'écoulement va modifier la valeur de ces fréquences, augmentant la première et diminuant la seconde pour aboutir à l'égalité des deux, condition propice à l'instabilité.

Cette première interaction permet donc à la deuxième d'entrer en jeu. Pour la comprendre, précisons d'abord que l'effort de portance est dirigé vers le haut lorsque l'avant de la voilure (le bord d'attaque) est dirigé vers le haut et inversement vers le bas lorsque le bord d'attaque est dirigé vers le bas. Dans notre cas, le phasage entre le battement de l'aile et sa torsion conduit à diriger la portance constamment dans la direction du mouvement. Le bord d'attaque est tordu vers le haut lorsque l'aile se fléchit vers le haut et il est tordu vers le bas lorsque l'aile se fléchit vers le bas. Dans ces conditions, la portance accentue indéfiniment le battement d'aile jusqu'à obtenir de très grandes déformations allant souvent jusqu'à la rupture. C'est un phénomène dit d'instabilité dynamique par couplage de fréquences.

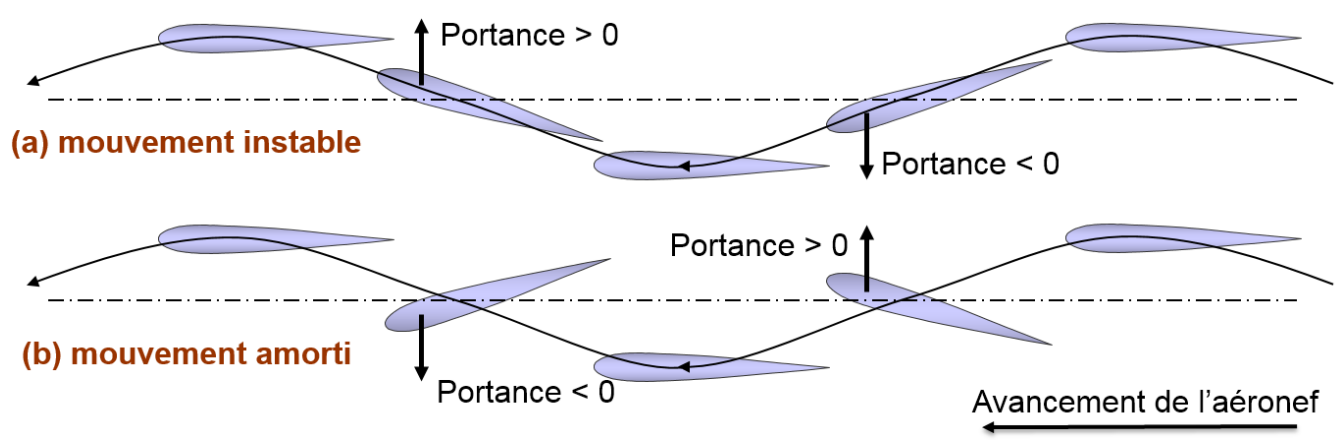

Encadré 1. Explication du phénomène de flutter. 


\section{Une brève histoire du flutter}

Le premier cas documenté de flutter dans l'histoire de l'aéronautique l'a été par F.W. Lanchester en 1916 [LAN 16] et concernait les empennages du bombardier biplan Handley Page 0/400. L'entre-deux guerres a été une période très active pour cette discipline du fait de l'augmentation de la vitesse de vol des aéronefs suivant un rythme plus soutenu que celui des progrès dans le domaine de la rigidité des structures. Plusieurs cas dramatiques ont notamment été rencontrés dans les années 20 et 30 lors de compétitions sur des voilures d'avions de vitesses ("Air Racers ») comme le Curtis R6 en 1924 ou le Gee Bee en 1931 [GAR 81]. Le type d'instabilités rencontrées après la deuxième guerre mondiale a évolué et concerne davantage des problèmes liées aux effets de Mach survenant proche du mur du son ainsi qu'à des interactions avec des emports externes (armements, réservoirs,...). La NACA, ancêtre de la NASA, a relevé 54 incidents liés au flutter impliquant des aéronefs militaires américains sur la période 1947-1956. En France, un accident dramatique impliquant un flutter de l'empennage horizontal est survenu lors d'un vol d'essai du chasseur Mirage F1 le 18 mai 1967 qui a entraîné la mort du pilote René Bigand.

\section{Encadré 2. Historique du phénomène de flutter.}

Ces trois accidents et les échecs de ces programmes nous font comprendre la difficulté de mise en œuvre de tels engins. La faible énergie disponible oblige les concepteurs à créer une voilure à la fois très grande et très légère donc nécessairement très souple. Ces engins ne peuvent être conçus en optimisant de manière séparée chaque discipline, c'est-à-dire l'aérodynamique, la structure et la dynamique du vol, mais imposent de considérer les phénomènes physiques que peuvent entraîner le mélange de ces disciplines. Or ces derniers sont encore mal connus quand les voilures deviennent très souples et de nombreux travaux de simulation sont en cours à travers le monde depuis le début des années 2000 [SHE 07 ; PAL 10 ; KIR 17].

\subsection{Une voie alternative pour remédier au flutter : le tissage aéroélastique}

Des solutions pour repousser les vitesses critiques d'apparitions de ces phénomènes dangereux voire mortels ont rapidement été trouvées par les ingénieurs aéronautiques. Elles sont basées sur la modification de deux paramètres principaux, à savoir la répartition des masses d'une part et la rigidification de la structure d'autre part. L'idée dans le deux cas est de modifier les fréquences propres de flexion et de torsion de la voilure en vol (encadré 1).

Le point commun de ces deux solutions est de s'opérer au détriment de la masse totale de l'aéronef et donc au final au détriment de son endurance. Il apparaît donc nécessaire pour concevoir des aéronefs à endurance maximale de développer des solutions alternatives permettant d'assurer une plage de vitesse de vol compatible avec le domaine d'emploi qui soit exempte d'instabilités et ce sans avoir recours à des méthodes alourdissant la structure. Un effort particulier est également à fournir dans le domaine de la modélisation de ces phénomènes d'interaction au regard de la complexité des mécanismes d'instabilité récemment rencontrés.

Parmi ces solutions alternatives, on peut citer le tissage aéroélastique (aeroelastic tailoring), technique qui consiste à exploiter les spécificités des matériaux composites. Ces matériaux, dits stratifiés, sont composés de couches successives de fibres de verre ou de carbone orientées dans des directions particulières le tout étant plongé dans une résine polymère, le plus souvent époxy. Suivant l'orientation successive de ces couches de fibre, on peut faire émerger du matériau final des couplages entre ces différents modes de déformations.

Appliqué à une aile on peut ainsi coupler la déformation en flexion (battement de l'aile) à la déformation en torsion. On voit donc ici apparaître un moyen de contrarier la tendance naturelle des 
fréquences en battements et en torsion de l'aile à se rejoindre lorsque la vitesse de vol augmente et ce faisant repousser les vitesses critiques d'apparition du flutter.

Cette technique présente deux avantages majeurs. D'abord elle n'a pas ou peu d'impact sur le bilan de masse de la voilure finale. Ensuite c'est une technique dite " passive » dans le sens où elle ne met pas en œuvre d'actionneur mécanique ou électrique et s'affranchit donc d'un risque de panne. Cette technique a été initiée dans les années 70 sur l'avion expérimental à flèche inverse américain X29 [PUT 84] (figure 9). Sa transposition au cas des voilures très allongées et très souples de drone solaire à haute altitude est aujourd'hui à l'étude. Elle s'avère complexe car comme évoqué dans le rapport d'accident du drone Helios [NOL 04], la physique qui est en jeu se caractérise par un fort couplage entre les différentes disciplines que sont l'aérodynamique, la mécanique des structures, la mécanique du vol et les lois de commandes de vol automatiques.

L'étude de ces phénomènes complexes est donc par essence fortement transdisciplinaire et elle est encore inaccessible à des outils de simulation numérique haute-fidélité en raison d'un coût en temps de calcul prohibitif. Un effort de recherche conséquent est porté sur la mise au point de modèles simplifiés pertinents capable à la fois de modéliser les couplages générés par le tissage aéroélastique et de prédire finement les vitesses d'apparitions des instabilités comme le flutter [KIR 17].

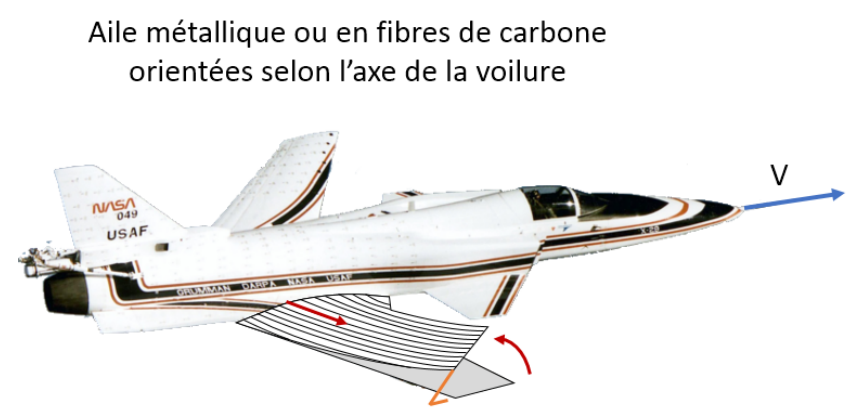

L'aile fléchit et se vrille vers l'arrière > instable

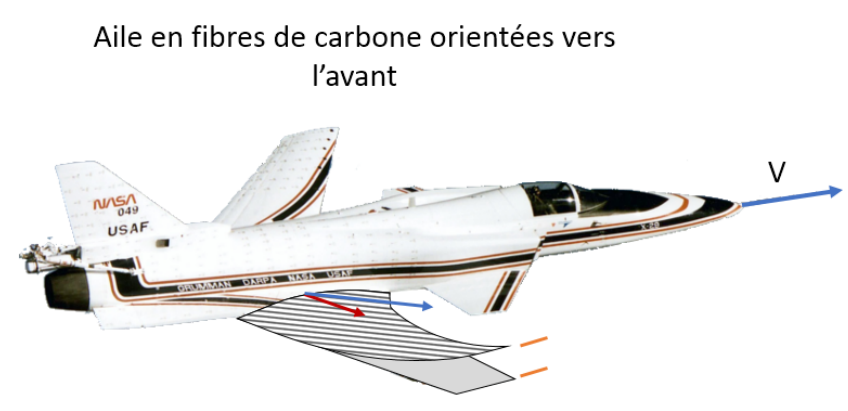

L'aile fléchit mais ne se vrille pas > stable

Figure 9. Explication du principe du tissage aéroélastique sur l'aile du X-29 de la NASA

Ces axes de recherches dépassent d'ailleurs le cadre restrictif des drones solaires haute altitude puisqu'il représente une voie d'optimisation en aéronautique commerciale. L'idée, soutenue notamment à travers le programme européen horizon 2020 Flexop [FLE 15], est de concevoir des ailes de plus grande envergure sans augmenter le bilan de masse et donc plus souples tout en garantissant un domaine de vol exempt d'instabilités aéroélastiques. Le tissage aéroélastique est un exemple de brique technologique en cours de développement pour atteindre cet objectif, il en existe d'autres comme des méthodes actives utilisant des matériaux piézoélectriques pour contrôler la déformation des surfaces portantes.

\section{Conclusion}

Que ce soit à travers une solution de drone solaire à voilure fixe ou de plus léger que l'air, la course à l'endurance est un puissant moteur pour la recherche et l'innovation, notamment dans le domaine de l'aéroélasticité, les récents accidents étant là pour rappeler le chemin qui reste à parcourir. Ce défi scientifique et technologique a été présenté ici à travers le prisme de deux approches complémentaires que sont la transdisciplinarité et l'étude de solutions innovantes. Ces dernières sont au fondement de briques technologiques qui, ajoutées les unes aux autres, permettront à terme la concrétisation d'un concept né dans les années 1950 avec la découverte des cellules photovoltaïques.

Le cas des pseudo-satellites de haute altitude présenté dans cet article illustre parfaitement l'émulation et les synergies qui se créent autour d'un concept novateur entre la recherche scientifique et l'écosystème capable in fine de créer de la valeur [BAR 16]. Ainsi, les premiers succès obtenus, 
comme le vol du drone Zephyr 7 d'une durée de 14 jours, préfigurent la conquête d'un champ capacitaire quasi vacant situé entre les aéronefs conventionnels et les satellites. La mise au point de ces plateformes innovantes devrait permettre à terme d'apporter des solutions adaptables et réactives pour des applications civiles et militaires, aussi bien dans le domaine de l'observation que des télécommunications.

\section{Bibliographie}

[BAR 16] BARbaroux, P., ATtour, A., SchenK, E., Knowledge Management and Innovation: Interaction, Collaboration, Openness. ISTE publishing, 130 pages, 2016.

[BAU 15] Baurreau F., Staraj R., Ferrero F., Lizzi L., Ribero J. M., \& Chessel J. P., "Stratospheric platform for telecommunication missions". In Antennas and Propagation \& USNC/URSI National Radio Science Meeting, IEEE International Symposium, pp. 914-915, 2015.

[BOU 85] BOUCHER R. J., "Sunrise, the World's First Solar-Powered Airplane", Journal of Aircraft, 1985, vol. 22, p. 840-846.

[CES 06] CESTINO E., "Design of solar high altitude long endurance aircraft for multi payload \& operations". Aerospace science and technology, 10(6), 541-550, 2006.

[GAR 81] GARRICK I. E. \& REED W. H., "Historical development of aircraft flutter", Journal of Aircraft, vol. 18, no 11, p. 897-912, 1981.

[FLE 15] "FLEXOP”. [En ligne]. Disponible sur: https://www.flexop.eu/index.html. [Consulté le: 20-nov-2017].

[FLI 98] FLITTIE K. \& CURTIN B., "Pathfinder solar-powered aircraft flight performance”. In 23rd Atmospheric Flight Mechanics Conference, paper 4446, 1998.

[HER 02] Herwitz S., Johnson L., Arvesen J., Higgins R., Leung J., \& DunAGAN S. (2002). "Precision agriculture as a commercial application for solar-powered unmanned aerial vehicles". In 1st AIAA UAV Conference, Portsmouth, paper 3404, 2001.

[JUV 16] JUVÉ L., FOSSE J., JOUBERT E., \& FOUQUET N., “Airbus Group electrical aircraft program, the E-FAN project”. In 52nd AIAA/SAE/ASEE Joint Propulsion Conference, p. 4613, 2016.

[KIR 17] Kirsch B., MONTAGNIER O., BENARD E., \& FAURE T., "Effet de l'anisotropie des composites sur le comportement aéroélastique des drones HALE », in Journées Nationales sur les Composites 2017, Champs-Sur-arne, 2017.

[LAN 16] LANCHESTER F. W., "Torsional Vibrations of the Tail of an Aeroplane", Aeronaut Res. Com R M, vol. 276, 1916.

[MCC 83] MACCREAdY P. B., Lissaman P. B. S., Morgan W. R., \& BuRke J. D., "Sun-powered aircraft designs". Journal of Aircraft, 20(6), 487-493, 1983.

[MON 09] MONTAGNIER O. \& BOVET L., "Optimisation of a solar-powered high altitude long endurance uav with composite wings". In Proceedings of the 3rd EUropean Conference for AeroSpace Sciences, 2009.

[MON 10] MONTAGNIER O. \& Bovet L., “Optimization of solar powered HALE UAV”, in Proc. 2nd Int. Congress of the Aeronautics Science, ICAS 2010, Cancun, 2010.

[MON 13] MONTAGNIER O., "Drones solaires : la quête du vol perpétuel”, dans S. MAZOYER, J. DE LESPINOIS, E. GOFFI, G. Boutherin, C. PAJON, Les drones aériens : passé, présent et avenir. Approche globale, La Documentation française, Paris, pp. 491-501, 2013.

[NOL 04] Noll T. E., Brown J. M., Perez-Davis M. E., Ishmael S. D., Tiffany G. C. \& GAIER M., "Investigation of the Helios prototype aircraft mishap", NASA Report, vol. 9, 2004.

[NOT 08] Noth, A., "Histoire de l'Aviation Solaire”, Laboratoire des Systèmes Autonomes, ETH Zürich, Suisse, 2008.

[NTS 17a] "NTSB report Aquila". [En ligne]. Disponible sur:

https://www.ntsb.gov/_layouts/ntsb.aviation/brief.aspx?ev_id=20160701X62525\&key=1. [Consulté le: 20-nov-2017].

[NTS 17b] "NTSB report Solara 50" [En ligne]. Disponible sur: https://www.ntsb.gov/_layouts/ntsb.aviation/brief.aspx?ev_id=20150505X85410. [Consulté le: 20-nov-2017].

[PAL 10] PALACIOS, R., MURUA, J., COOK, R., "Structural and aerodynamic models in nonlinear flight dynamics of very flexible aircraft", AIAA Journal, 48(11):2648-2659, 2010. 
[PUT 84] T. W. PUTNAM, “X-29 flight-research program”, 1984.

[ROM 13] RomeO, G., Borello, F., CORREA, G., \& CESTINO, E., "ENFICA-FC: Design of transport aircraft powered by fuel cell \& flight test of zero emission 2-seater aircraft powered by fuel cells fueled by hydrogen", International journal of hydrogen energy, 38(1), 469-479, 2013.

[SHE 07] SHEARER, C., CESNIK, C., "Non-linear flight dynamics of very flexible aircraft." Journal of Aircraft, vol. 44, no 5, p.1528-1545, 2007. 